\title{
Cell biology and immunology lessons taught by Legionella pneumophila
}

\author{
Wenhan Zhu \& Zhao-Qing Luo* \\ Department of Biological Sciences, Purdue University, West Lafayette, IN47907, USA \\ Received July 27, 2015; accepted August 27, 2015; published online November 20, 2015
}

\begin{abstract}
Legionella pneumophila is a facultative intracellular pathogen capable of replicating within a broad range of hosts. One unique feature of this pathogen is the cohort of ca. 300 virulence factors (effectors) delivered into host cells via its Dot/Icm type IV secretion system. Study of these proteins has produced novel insights into the mechanisms of host function modulation by pathogens, the regulation of essential processes of eukaryotic cells and of immunosurveillance. In this review, we will briefly discuss the roles of some of these effectors in the creation of a niche permissive for bacterial replication in phagocytes and recent advancements in the dissection of the innate immune detection mechanisms by challenging immune cells with $L$. pneumophila.
\end{abstract}

Type IV secretion, inflammasome, effectors, posttranslational modification, vesicle transport

Citation: Zhu, W., and Luo, Z. (2016). Cell biology and immunology lessons taught by Legionella pneumophila. Sci China Life Sci 59, 3-10. doi: $10.1007 / \mathrm{s} 11427-015-4945-\mathrm{x}$

\section{Legionella AND THE DOT/ICM TYPE IV SECRETION SYSTEM}

The discovery of Legionella pneumophila dates back to 1976, when a mysterious disease affected 221 American veterans attending the American Legion Convention in Philadelphia. The causative agent for this highly concerning outbreak was identified three year later and was designated as Legionella pneumophila, a gram-negative bacterium that belongs to the $\gamma$-proteobacteria (McDade et al., 1977). Subsequent studies revealed that L. pneumophila is an intracellular pathogen capable of replicating in highly diverse hosts, ranging from fresh water amoebae to mammalian alveolar macrophages (Stone et al., 1999). One salient feature associated with L. pneumophila infection is that the bacterial phagosome does not undergo the default phagosomal matu-

*Corresponding author (email: luoz@purdue.edu)

†Current address: Department of Microbiology, UT Southwestern Medical Center, Dallas, TX 75390, USA ration process characterized by fusion with the lysosomal network, particularly in the early phase of infection; rather it develops into an organelle permissive for intracellular bacterial replication termed the Legionella-containing vacuole (LCV) (Ensminger and Isberg, 2009; Horwitz, 1983; Tilney et al., 2001) (Figure 1). The capability of this bacterium to construct such a unique niche is mostly attributed to the activity of effectors translocated by the Dot/Icm type IV secretion system (Huang et al., 2011; Isberg et al., 2009; Lifshitz et al., 2013; Luo and Isberg, 2004; Xu and Luo, 2013; Zhu et al., 2011). These effectors function to re-orchestrate various host processes to allow intracellular bacterial growth. The infection biology of L. pneumophila provides an excellent example of the sophistication of the interactions between bacterial pathogens and their hosts. Further, investigations of the sensing of the Dot/Icm translocated proteins and/or their activity by immune cells such as macrophages has allowed the identification of novel immunosurveillance mechanisms often not seen in infections with more adapted pathogens. 


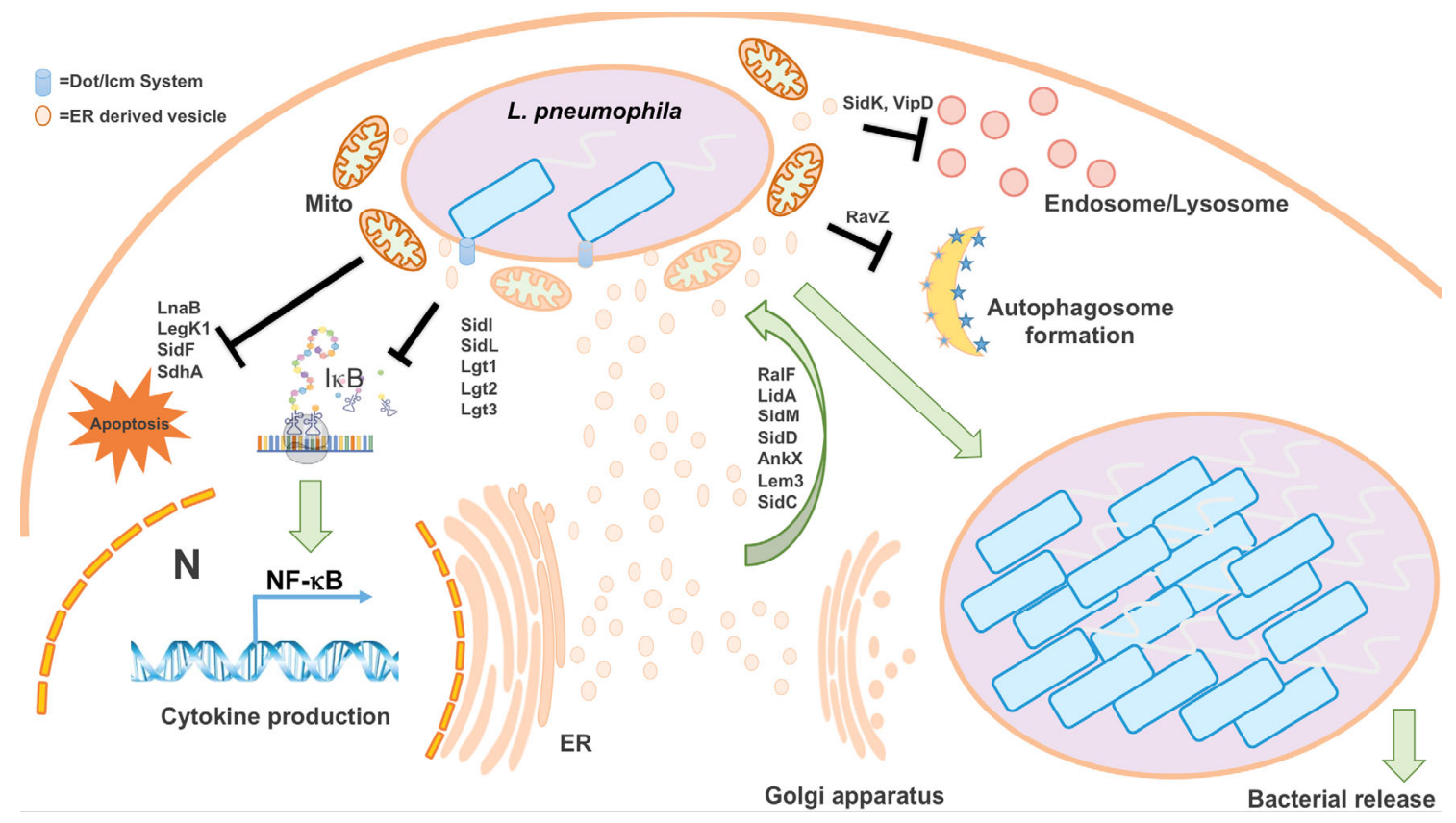

Figure 1 Modulation of multiple host processes by Dot/Icm effectors. The formation of the LCV containing numerous L. pneumophila cells in permissive macrophages requires active modulation of multiple host processes by a large cohort of effectors. The events shown in this Figure include cell death, activation of the NF- $\mathrm{KB}$ pathway, membrane transport between the ER and the Golgi apparatus, autophagy and the default phagosome maturation pathway that delivers newly formed phagosomes to the lysosomal network. Dot/Icm effectors involved in these processes were listed individually or as groups.

\section{MODULATION OF HOST PROCESSES BY DOT/ICM EFFECTORS}

L. pneumophila actively modulates host processes at both transcriptional and posttranslational levels. For example, infection with virulent L. pneumophila induces many anti-apoptotic genes via the activation of the NF-кB pathway (Abu-Zant et al., 2007; Losick and Isberg, 2006); effectors such as LegK1 and LnaB are involved in such activation (Ge et al., 2009; Losick et al., 2010). Histone methylation by RomA/LegAS4 causes the repression of innate immune genes and the activation of rDNA transcription, respectively (Li et al., 2013; Rolando et al., 2013), which are important to intracellular replication. Both the inhibition of protein synthesis (Belyi et al., 2006; Fontana et al., 2011) and the induction of cell death (via the activation of caspase 3 ) are mediated by multiple effectors (Zhu et al., 2013), most likely at the posttranslational level. Similar to many intracellular pathogens, L. pneumophila also targets components of the host actin network (Franco et al., 2012; Guo et al., 2014; Michard et al., 2015), likely to facilitate the biogenesis of the LCV by finely modulating the function of the actin cytoskeleton.

The rapid conversion of the bacterial phagosome into an organelle resembling the endoplasmic reticulum (ER) is a unique feature associated with L. pneumophila infection; this process involves the function of many effectors, some of which target important signaling hubs of host membrane trafficking. Phosphatidylinositols (PIs) are essential in defining organelle identity and in signaling cascades in vesicle trafficking and actin cytoskeleton structure (Di Paolo and De Camilli, 2006; Odorizzi et al., 2000). L. pneumophila harnesses the metabolism and cellular distribution of PIs using specific enzymes. On one hand, this bacterium injects a PI(3)P phosphatase into host cells (Toulabi et al., 2013), which may together with Rab5-activated VipD (Gaspar and Machner, 2014), function to remove PI(3)P from the surface of specific organelles (including the LCV), thus preventing the fusion of the LCV with the lysosomal network. On the other hand, L. pneumophila actively enriches PI(4)P on the LCV by employing enzymes of both bacterial and host origins. The PI-3-phosphatase SidF produces PI(4)P from $\mathrm{PI}(3,4) \mathrm{P} 2$ directly or from $\mathrm{PI}(3,4,5) \mathrm{P} 3$ together with OCRL, a host 5-phosphatase (Hsu et al., 2014). The host phosphatidylinositol-4-kinase III $\alpha$ (PI4KIII $\alpha$ ) also contributes to such enrichment (Hubber et al., 2014). The PI(4)P on the LCV allows the anchoring of PI(4)P-binding effectors such as SidM/DrrA, SidC and SdcA, which may compartmentalize the activity of these effectors to the surface of the LCV (Brombacher et al., 2009; Ragaz et al., 2008; Weber et al., 2006). Enriched PI(4)P may also mimic the lipid composition of the cis-Golgi compartment (Di Paolo and De Camilli, 2006; Odorizzi et al., 2000), making the LCV a "natural" recipient compartment for the incoming ER-derived vesicles. L. pneumophila also interferes with the biosynthesis of diacylglycerol (DAG) and phosphatidic acid (PA) in host cells (Viner et al., 2012) but the importance of such manipulation is not clear.

Concomitant with its interference with host lipid metabolism, L. pneumophila remodels the LCV membrane by hi- 
jacking the activity of key regulators of membrane trafficking such as Arf1 (Nagai et al., 2002) and Rab1 (Machner and Isberg, 2006), the small GTPases essential for vesicle transport between the ER and the Golgi apparatus. At least five Dot/Icm effectors completely control the activity of this regulatory protein. Amongst these, SidM/DrrA is a multifunctional protein; it anchors to the LCV surface by binding to PI(4)P and activates Rab1 with its guanine nucleotide exchange factor (GEF) activity (Machner and Isberg, 2006; Murata et al., 2006) and locks the GTPase in its active form with its AMPylating (adenylyl transferase) activity (Muller et al., 2010). Following the removal of the AMPylation modification by SidD (Neunuebel et al., 2011; Tan and Luo, 2011), Rab1 is inactivated by LepB, a bacterial GTPase activation protein (GAP) (Ingmundson et al., 2007). In murine macrophages, these proteins exert a temporal regulation of Rab1 activity on the LCV in the first 4 hours after bacterial uptake (Neunuebel et al., 2011; Tan and Luo, 2011) (Figure 1). The complexity of Rab1 activity regulation is aggregated by the phosphorylcholine transferase AnkX (Mukherjee et al., 2011; Tan et al., 2011) and Lem3, an enzyme that reverses the modification imposed by AnkX (Tan et al., 2011). It is possible that AnkX targets Rab1 at the cis-Golgi compartment to block the fusion of ER-derived vesicles, which may facilitate the re-direction of such vesicles to the LCV. In this model, the role of Lem3 is to safeguard (accidental) AnkX-mediated inhibition of Rab1 activity on the LCV. The bacterium also targets the Ran small GTPase to regulate microtubule polymerization, motility of its phagosome and infected cells (Rothmeier et al., 2013; Simon et al., 2014).

Dot/Icm effectors also target other cellular events important for vesicle transport such as autophagy (Choy et al., 2012) and the v-ATPase (Xu et al., 2010), which regulates membrane fusion by controlling the $\mathrm{pH}$ and other physiological features of vacuoles. Finally, re-orchestration of the host ubiquitination pathways is emerging as an important branch of the bacterium's strategy. At least eight Dot/Icm effectors appear to possess F-box or U-box domains typical of known E3 ligases (Hubber et al., 2013). Among these, LegU1, LegAU13/AnkB and LubX have been demonstrated to possess E3 ligase activity (Ensminger and Isberg, 2010; Kubori et al., 2008). SidC and SdcA are recently discovered unique E3 ligases important for efficient enrichment of both ubiquitinated species and proteins of ER origin onto the bacterial phagosome (Hsu et al., 2014). Interestingly, Rab1 is monoubiquitinated during L. pneumophila infection in a manner that requires SidC/SdcA (Horenkamp et al., 2014), yet these two E3 ligases do not detectably ubiquitinate this small GTPase in vitro or in cells transfected to express these proteins (Hsu et al., 2014). Future identification of the protein substrates of these E3 ligases will provide exciting insights into the signaling cascades that potentially link ubiquitination to vesicle transport and other cellular events important for the biogenesis of the LCV.

\section{IMMUNE RECOGNITION IN L. pneumophila INFECTION}

\section{Extracellular immune recognition}

Most individuals susceptible to L. pneumophila infection are immunocompromised (Kumpers et al., 2008), suggesting that this bacterium does not effectively evade immune detection. The immune system recognizes L. pneumophila by employing an arsenal of germ-line coded Pattern-Recognition-Receptor (PRRs) distributed on different cellular locales to perceive the presence of associated Pathogen-Associated-Molecular-Pattern (PAMPs), or by detecting alterations of the cellular activities imposed by the bacterium (Abt et al., 2012)

The PRRs are categorized based on the subcellular location where the receptor initiates the signaling. Toll-like-receptors (TLRs) and C-type lectins are among the most well-characterized membrane associated receptors that mainly function to recognize extracellular ligands (some TLRs are located in endosomes that recognize intracellular ligands) (Lee and Kim, 2007). These receptors recognize a wide range of ligands. For example, mouse codes for 13 TLRs, and the ligands to these receptors includes bacterial lipopolysaccharides (LPS), peptidoglycans, triacyl lipopeptides, and flagellin, as well as the zymosan of fungi (Broz and Monack, 2013). It has been demonstrated that TLRs are essential to mounting appropriate immune responses to $L$. pneumophila, as mice lacking MyD88, the signaling adaptor shared by all TLRs except TLR3, fail to clear L. pneumophila in the lung and eventually succumb to acute pulmonary infection, with bacteria spreading to lymph nodes and the spleen (Archer and Roy, 2006). Among TLRs, TLR2, TLR4 and TLR5 are the major contributors to the recognition of $L$. pneumophila during infection, as the lack of TLR2 enhances bacterial growth in a mouse model, and the polymorphisms of TLR4 and TLR5 are associated with resistance to Legionnaires' disease (Archer et al., 2009; Hawn et al., 2005; Hawn et al., 2003).

\section{Intracellular immune recognition}

Extracellular PRRs alone are not sufficient in defending against microbial infections, especially those by intracellular pathogens. It is therefore not surprising that mammals code for a large cohort of cytoplasmic PRRs, including the NOD-like receptors (NLRs), pyrin and HIN domain containing family (PYHIN), RIG-I-like receptors (RLRs) and numerous cytosolic nucleic acid sensors to guard the cytosol from invading microbes (Broz and Monack, 2013). Some NLRs upregulate the transcription of downstream signaling components, such as the NF- $\kappa \mathrm{B}$ and the IFN- $\beta$ production pathways upon engaging their ligands (Strober et al., 2006). Other NLRs, however, drive the assembly of large protein complexes termed inflammasomes, which cause pyroptotic cell death and the secretion of mature inflammatory cytokines, such as IL-1 $\beta$ and IL-1 $\alpha$ (Lamkanfi and Dixit, 2014). 
The diverse ligand recognition capacity of NLRs makes them one of the most important guardians of the cytoplasm. For example, NLRP1b responds to the protease activity of the Bacillus anthracis lethal toxin and to Toxoplasma gondii infection (Cirelli et al., 2014), whereas the AIM2 inflammasome responds to cytosolic DNA molecules (Rathinam et al., 2010). Similarly, the NLRC4 inflammasome responds to many bacterial PAMPs, such as the rod and needle proteins of bacterial Type III secretion system as well as flagellin (Zhao et al., 2011). The capability of NLRC4 to recognize such a wide repertoire of PAMPs is attributed to the NAIP proteins (also called baculoviral IAP repeat-containing protein), which serve as adaptors to bridge the interaction between NLRC4 and the PAMP molecules (Zhao et al., 2011). So far, the NLRP3 inflammasome is known to respond to the most diverse PAMPs, which include bacterial, fungal and viral pathogens, as well as pore formation toxins, protein amyloid, extracellular ATP, and inorganic substances such as silica and alum (Lamkanfi and Dixit, 2014).

The discovery of the intracellular sensor for flagellin is largely attributed to the research aiming at understanding the inability of macrophages from most of the inbred mouse strains to support intracellular L. pneumophila replication (Yamamoto et al., 1988). For example, BMDMs (Bone Marrow Derived Macrophage) from C57BL/6 mouse effectively clear L. pneumophila, and the bacterial clearance is accompanied by extensive pyroptosis (Derre and Isberg, 2004), suggesting the involvement of active host immune responses. However, L. pneumophila successfully replicates in macrophages from A/J mice (Yamamoto et al., 1988). Taking advantage of the sharply different responses of the two mouse strains to L. pneumophila, Dietrich et al. mapped the genetic elements responsible for the permissiveness of $\mathrm{A} / \mathrm{J}$ mice to the Lgn1 locus, which was later pinpointed to the gene Naip5 (Diez et al., 2003). The very same phenotype also was utilized to identify flagellin as the bacterial factor that engages Naip5 (Molofsky et al., 2006; Ren et al., 2006). Upon engaging flagellin, Naip5 recruits and activates NLRC4, which recruits and activate caspase-1(Kofoed and Vance, 2011; Zhao et al., 2011), leading to the secretion of inflammatory cytokines (IL-18 and IL-1 $1 \beta$ ) and pyroptotic cell death. These events eventually lead to further recruitment of other immune cells and the removal of the replicative niche, explaining the resistance to L. pneumophila infection by most mouse lines.

In permissive mouse macrophages, L. pneumophila maintains its lifestyle as a vacuolar pathogen. The stability of LCV membrane is affected by the lipase PlaA, which seems to destabilize the vacuole (Creasey and Isberg, 2012). Interestingly, the effects of PlaA are counteracted by the effector SdhA, which maintains the integrity of the LCV, very likely by modulating host membrane trafficking (Creasey and Isberg, 2012). L. pneumophila lacking SdhA aberrantly enters the cytosol, leading to the activation of the AIM-2 inflammasome and the noncanonical caspase-11 inflammasome (Aachoui et al., 2013; Ge et al., 2012). These phenomena have facilitated the discovery of the caspase- 11 inflammasome, which was later shown to be activated directly by intracellular LPS (Aachoui et al., 2013; Shi et al., 2014). These exciting successes in revealing previously underappreciated immune pathways by studying $L$. pneumophila infection clearly demonstrate the great potential of investigating host responses using less adapted pathogens (Figure 2).

Other cytoplasmic PRRs such as RLRs, including retinoic acid-inducible gene I (RIG-I), LGP2 and the melanoma differentiation-associated protein 5 (MDA5), detect nucleic acid and their derivatives, which are one of most important categories of MAMPs (Broz and Monack, 2013). RIG-I, for example, recognizes cytoplasmic dsRNA to promote the prion conversion of MAVS (Mitochondrial antiviralsignaling protein), which signals to activate the production of IFN- $\beta$ (Cai et al., 2014). Of particular interest is the long-anticipated revelation of the major cytoplasmic dsDNA sensor, cGAS (Cyclic GMP-AMP synthase) (Sun et al., 2013). This sensor is special as it is an enzyme itself, which catalyzes the formation of cyclic di-GMP-AMP upon recognizing dsDNA. The cyclic di-GMP-AMP is then sensed by STING (STimulator of INterferon Genes), which signals the production of type I IFN (Burdette et al., 2011). L. pneumophila can also be recognized by the AIM2 inflammasome, probably by bacterial DNA "leaked" into the host cytosol by the Dot/Icm system (Berrington et al., 2010) (Figure 2). Since L. pneumophila infection also induces Type I Interferon production in a STING-(stimulator of interferon genes) dependent manner, it is tempting to postulate that the "leaked" bacterial DNA also engages the cGAS (cyclic GMP-AMP synthase)-c-di-AMP-GMP-STING pathway (Ge et al., 2012; Lippmann et al., 2011; Monroe et al., 2009) (Figure 2). Alternatively, bacterial cyclic di-GMP-AMP may reach host cytosol via the Dot/Icm system or in conjunction with other transporters, akin to that observed in Listeria monocytogenes (Crimmins et al., 2008; Woodward et al., 2010).

In addition to pyroptosis, intracellular surveillance of foreign molecules causes permeabilization of the lysosome, an organelle that is emerging as a central signaling hub for various pathological stresses, including stroke, acute pancreatitis, parasitic and infections (Aits and Jaattela, 2013). Upon perceiving these stresses, lysosomal membranes undergoes selective or complete permeabilization, leading to the release into the cytosol, of its contents, including proteases of the cathepsin family (Aits and Jaattela, 2013). Cathepsins are capable of inducing cell death of various forms (Turk and Turk, 2009). A recent discovery reveals that $L$. pneumophila "accidently" delivers RpsL, a conserved bacterial ribosomal protein into the cytosol, where it causes lysosomal membrane permeabilization (LMP), presumably by activating a signaling cascade upon engaging a yet to be identified receptor (Zhu et al., 2015). Cathepsins such ca- 


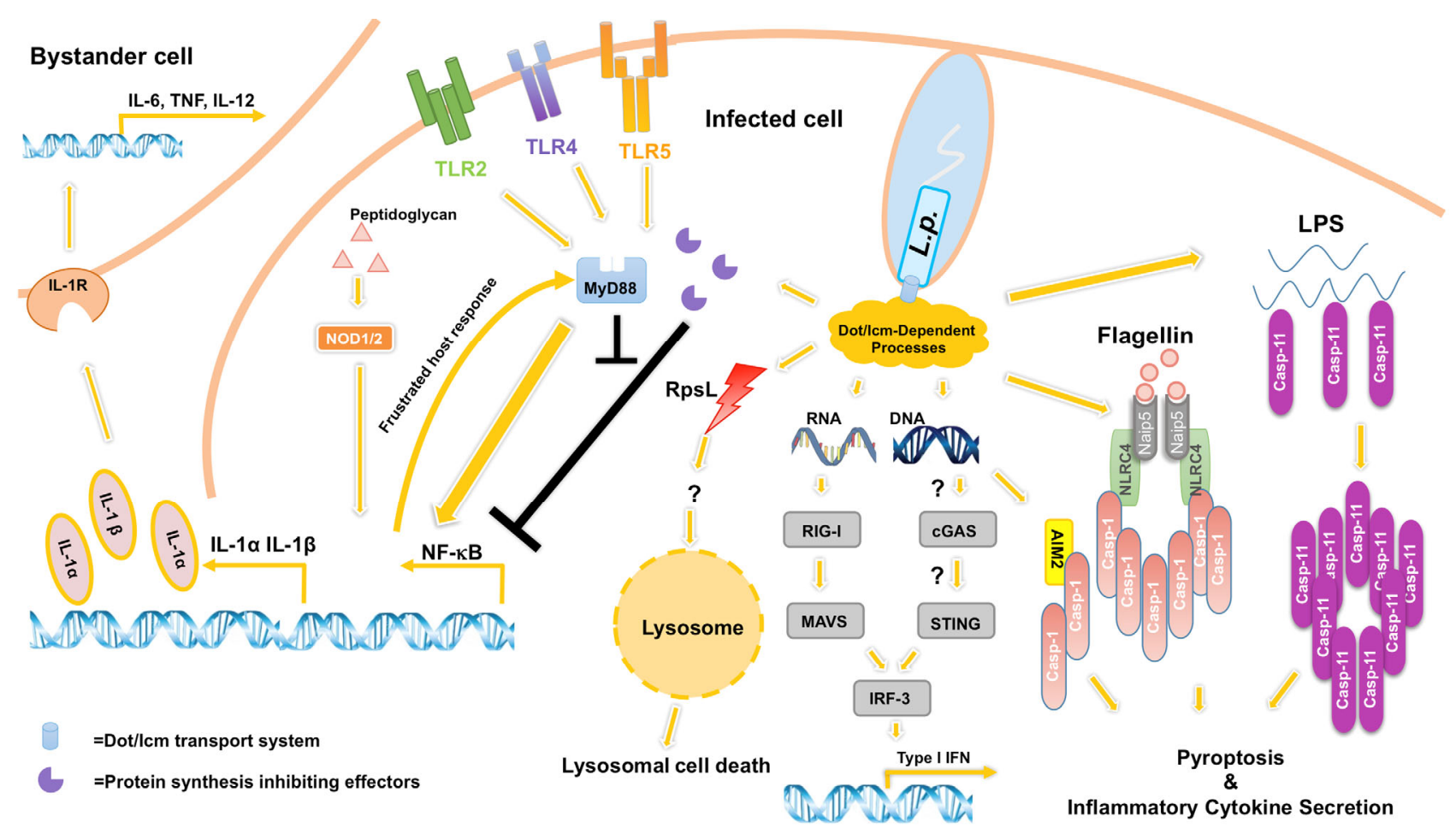

Figure 2 Immune recognition of L. pneumophila. Interactions between L. pneumophila and macrophages activate immune responses at both transcriptional and posttranslational levels. The most notable events are those that occur in response to the activity of the Dot/Icm transporter, including the production of type I interferon, the activation of various inflammasomes and the induction of lysosomal membrane permeabilization (LMP). AIM2, Absent in melanoma 2; Casp-1, capase-1; Casp-11, caspase-11; cGAS, cyclic di-GMP-AMP synthase; IFN, Interferon; IRF-3, Interferon regulatory factor 3; LPS, lipopolysaccharide; MAVS, mitochondrial antiviral-signaling protein; MyD88, Myeloid differentiation primary response gene 88; Naip5, NLR family, apoptosis inhibitory protein 5; NF- $\mathrm{BB}$, nuclear factor kappa-light-chain-enhancer of activated B cells; NLRC4, NLR family CARD domain-containing protein 4; NOD1/2, Nucleotide-binding oligomerization domain-containing protein 1 and 2; RIG-I, retinoic acid-inducible gene 1; STING, stimulator of interferon genes; TLR2, Toll like receptor 2; TLR5, Toll like receptor 5 .

thepsin B and cathepsin D are released in response to infections with $L$. pneumophila expressing wild type RpsL or artificially delivered protein (Zhu et al., 2015). More interestingly, a Lys88Arg mutation that also confers resistance to the antibiotic streptomycin, allows the bacterium to replicate in mouse macrophage (Zhu et al., 2015), suggesting the recognition of the same epitope by antibiotics and the immune system.

\section{IMMUNITY TRIGGERED BY L. pneumophila EFFECTORS}

PAMPs are not the only microbe-associated features sensed by that the immune systems are sensing. The observation that different cultivars of the same plant respond distinctively to different pathogen races has prompted plant pathologists to postulate the "gene-for-gene" hypothesis (Flor, 1971), which suggests that resistant plants code for the "resistant gene (R gene)" that is required for the recognition of the virulence factor "avirulence gene ( $a v r$ gene)". Since the presence of the avr gene is sufficient to trigger immune responses from resistant plants, it was proposed that the $\mathrm{R}$ gene product directly recognizes the gene product of the Avr, which is similar to the PRR-PAMP paradigm in metazoans (Stuart et al., 2013). However, as the interactions between Avr and R genes were rarely demonstrated, and that the Avr genes usually codes for bacterial virulence factors with distinct biochemical activities, Dangl and other researchers hypothesized that $\mathrm{R}$ gene products are not recognizing the Avr proteins per se, but instead their biochemical consequences (Chisholm et al., 2006). This hypothesis was supported by the observation that Avr proteins lacking their enzymatic activities are not immunogenic (Jones and Dangl, 2006). Thus, the immunity triggered by perceiving the biochemical consequences of bacterial effectors is defined as effector-triggered immunity (ETI) (Jones and Dangl, 2006). Similar hypothesis called "pattern of pathogenesis" was proposed for the mammalian system (Jones and Dangl, 2006). It is now clear that ETI exists in metazoan (Chisholm et al., 2006) as well. For instance, modification of the host protein RAC2 by the Escherichia coli CNF (cytotoxic necrotizing factor-1) activates the NF- $\kappa \mathrm{B}$ pathway (Boyer et al., 2011; Gurcel et al., 2006). Similarly, the pore formation activity of various bacterial toxins activates the NLRP3 inflammasome (Boyer et al., 2011; Gurcel et al., 2006) and the GAP activity of the Yersinia T3SS effector YopE triggers bacterial killing by macrophages (Wang et al., 2014). Finally, a recent study reveals that the Pyrin in- 
flammasome is an $\mathrm{R}$ gene equivalent in mammals, which senses various effector-mediated modifications on the small GTPase RhoA, including AMPylation, ADP-ribosylation, deamindation and glucosylation (Xu et al., 2014). Thus, ETI is an important branch of innate immune surveillance in metazoans.

ETI also contributes to the immune response to L. pneumophila infection. In macrophages, the MAP kinase pathway and the NF- $\mathrm{KB}$ pathway are highly induced by infections by wild type L. pneumophila but not by its dot/icm mutants (Abu-Zant et al., 2007; Losick and Isberg, 2006; Shin et al., 2008). Interestingly, protein synthesis inhibition conferred by at least five Dot/Icm substrates is part of the signals sensed by both the NF- $\mathrm{BB}$ and the MAP kinase pathways (Fontana et al., 2011; Fontana et al., 2012). Pro-longed activation of the NF- $\kappa \mathrm{B}$ pathway seen in infections with wild type $L$. pneumophila is achieved by delayed re-synthesis of $\mathrm{I} \kappa \mathrm{B}$, the labile inhibitory protein of NF- $\kappa \mathrm{B}$ (Fontana et al., 2011). The activation of the MAP kinase response by protein synthesis inhibition is multifaceted. First, many genes involved in this pathway are transcribed but not translated. The "frustrated" responses trigger signaling pathways to allow selective translation of at least two inflammatory cytokines, IL- $1 \alpha$ and IL- $1 \beta$, in a MyD88 dependent manner (Asrat et al., 2014). Analyses in a pulmonary infection mouse model suggest that, in vivo, bypassing the translational inhibition is mediated by the bystander cells not infected by L. pneumophila (Copenhaver et al., 2015).

In sum, the study of L. pneumophila pathogenesis in the past decade has greatly advanced our understanding of not only the mechanisms underlying the interactions between this pathogen and its hosts, but also the mechanisms in the biochemistry of posttranslational modifications, signaling and immune detection. Given the fact that the activity has only been assigned to a small fraction of the Dot/Icm substrates, it is anticipated that more excitement will be made in our analysis of the hundreds of effectors and the host response to these proteins in years to come.

Compliance and ethics The author(s) declare that they have no conflict of interest.

Acknowledgements We apologized to authors whose works cannot be cited here because of space limitation. The work on Legionella pathogenesis and immune response is supported by grants R56AI103168, K02AI085403 and R21AI105714 from the National Institutes of Health.

\section{References}

Aachoui, Y., Leaf, I.A., Hagar, J.A., Fontana, M.F., Campos, C.G., Zak, D.E., Tan, M.H., Cotter, P.A., Vance, R.E., Aderem, A., and Miao, E.A. (2013). Caspase-11 protects against bacteria that escape the vacuole. Science 339, 975-978.
Abt, M.C., Osborne, L.C., Monticelli, L.A., Doering, T.A., Alenghat, T., Sonnenberg, G.F., Paley, M.A., Antenus, M., Williams, K.L., Erikson, J., Wherry, E.J., and Artis, D. (2012). Commensal bacteria calibrate the activation threshold of innate antiviral immunity. Immunity 37 , $158-170$.

Abu-Zant, A., Jones, S., Asare, R., Suttles, J., Price, C., Graham, J., and Kwaik, Y.A. (2007). Anti-apoptotic signalling by the Dot/Icm secretion system of L. pneumophila. Cell Microbiol 9, 246-264.

Aits, S., and Jaattela, M. (2013). Lysosomal cell death at a glance. J Cell Sci 126, 1905-1912.

Archer, K.A., Alexopoulou, L., Flavell, R.A., and Roy, C.R. (2009). Multiple MyD88-dependent responses contribute to pulmonary clearance of Legionella pneumophila. Cell Microbiol 11, 21-36.

Archer, K.A., and Roy, C.R. (2006). MyD88-dependent responses involving toll-like receptor 2 are important for protection and clearance of Legionella pneumophila in a mouse model of Legionnaires' disease. Infect Immun 74, 3325-3333.

Asrat, S., Dugan, A.S., and Isberg, R.R. (2014). The Frustrated Host Response to Legionella pneumophila Is Bypassed by MyD88-Dependent Translation of Pro-inflammatory Cytokines. PLoS Pathog 10, e1004229.

Belyi, Y., Niggeweg, R., Opitz, B., Vogelsgesang, M., Hippenstiel, S., Wilm, M., and Aktories, K. (2006). Legionella pneumophila glucosyltransferase inhibits host elongation factor 1A. Proc Natl Acad Sci USA 103, 16953-16958.

Berrington, W.R., Iyer, R., Wells, R.D., Smith, K.D., Skerrett, S.J., and Hawn, T.R. (2010). NOD1 and NOD2 regulation of pulmonary innate immunity to Legionella pneumophila. Eur J Immunol 40, 3519-3527.

Boyer, L., Magoc, L., Dejardin, S., Cappillino, M., Paquette, N., Hinault, C., Charriere, G.M., Ip, W.K., Fracchia, S., Hennessy, E., Erturk-Hasdemir, D., Reichhart, J.M., Silverman, N., Lacy-Hulbert, A., and Stuart, L.M. (2011). Pathogen-derived effectors trigger protective immunity via activation of the Rac2 enzyme and the IMD or Rip kinase signaling pathway. Immunity 35, 536-549.

Brombacher, E., Urwyler, S., Ragaz, C., Weber, S.S., Kami, K., Overduin, M., and Hilbi, H. (2009). Rab1 guanine nucleotide exchange factor SidM is a major phosphatidylinositol 4-phosphate-binding effector protein of Legionella pneumophila. J Biol Chem 284, 4846-4856.

Broz, P., and Monack, D.M. (2013). Newly described pattern recognition receptors team up against intracellular pathogens. Nat Rev Immunol 13, 551-565.

Burdette, D.L., Monroe, K.M., Sotelo-Troha, K., Iwig, J.S., Eckert, B., Hyodo, M., Hayakawa, Y., and Vance, R.E. (2011). STING is a direct innate immune sensor of cyclic di-GMP. Nature 478, 515-518.

Cai, X., Chen, J., Xu, H., Liu, S., Jiang, Q.X., Halfmann, R., and Chen, Z.J. (2014). Prion-like polymerization underlies signal transduction in antiviral immune defense and inflammasome activation. Cell 156, 1207-1222.

Chisholm, S.T., Coaker, G., Day, B., and Staskawicz, B.J. (2006). Host-microbe interactions: shaping the evolution of the plant immune response. Cell 124, 803-814.

Choy, A., Dancourt, J., Mugo, B., O’Connor, T.J., Isberg, R.R., Melia, T.J., and Roy, C.R. (2012). The Legionella effector RavZ inhibits host autophagy through irreversible Atg8 deconjugation. Science 338, 1072-1076.

Cirelli, K.M., Gorfu, G., Hassan, M.A., Printz, M., Crown, D., Leppla, S.H., Grigg, M.E., Saeij, J.P., and Moayeri, M. (2014). Inflammasome sensor NLRP1 controls rat macrophage susceptibility to Toxoplasma gondii. PLoS Pathog 10, e1003927.

Copenhaver, A.M., Casson, C.N., Nguyen, H.T., Duda, M.M., and Shin, S. (2015). IL-1R signaling enables bystander cells to overcome bacterial blockade of host protein synthesis. Proc Natl Acad Sci USA 112, 7557-7562.

Creasey, E.A., and Isberg, R.R. (2012). The protein SdhA maintains the integrity of the Legionella-containing vacuole. Proc Natl Acad Sci USA 109, 3481-3486.

Crimmins, G.T., Herskovits, A.A., Rehder, K., Sivick, K.E., Lauer, P., Dubensky, T.W., Jr., and Portnoy, D.A. (2008). Listeria monocytogenes multidrug resistance transporters activate a cytosolic surveillance 
pathway of innate immunity. Proc Natl Acad Sci USA 105, 10191-10196.

Derre, I., and Isberg, R.R. (2004). Macrophages from mice with the restrictive Lgn1 allele exhibit multifactorial resistance to Legionella pneumophila. Infect Immun 72, 6221-6229.

Di Paolo, G., and De Camilli, P. (2006). Phosphoinositides in cell regulation and membrane dynamics. Nature 443, 651-657.

Diez, E., Lee, S.H., Gauthier, S., Yaraghi, Z., Tremblay, M., Vidal, S., and Gros, P. (2003). Bircle is the gene within the Lgn1 locus associated with resistance to Legionella pneumophila. Nature Genet 33, 55-60.

Ensminger, A.W., and Isberg, R.R. (2009). Legionella pneumophila Dot/Icm translocated substrates: a sum of parts. Curr Opin Microbiol 12, 67-73.

Ensminger, A.W., and Isberg, R.R. (2010). E3 ubiquitin ligase activity and targeting of BAT3 by multiple Legionella pneumophila translocated substrates. Infect Immun 78, 3905-3919.

Flor, H.H. (1971). Current status of the gene-for-gene concept. Phytopathology 45, 680-685.

Fontana, M.F., Banga, S., Barry, K.C., Shen, X., Tan, Y., Luo, Z.Q., and Vance, R.E. (2011). Secreted bacterial effectors that inhibit host protein synthesis are critical for induction of the innate immune response to virulent Legionella pneumophila. PLoS Pathog 7, e1001289.

Fontana, M.F., Shin, S., and Vance, R.E. (2012). Activation of host mitogen-activated protein kinases by secreted Legionella pneumophila effectors that inhibit host protein translation. Infect Immun 80, 3570-3575.

Franco, I.S., Shohdy, N., and Shuman, H.A. (2012). The Legionella pneumophila effector VipA is an actin nucleator that alters host cell organelle trafficking. PLoS Pathog 8, e1002546.

Gaspar, A.H., and Machner, M.P. (2014). VipD is a Rab5-activated phospholipase A1 that protects Legionella pneumophila from endosomal fusion. Proc Natl Acad Sci USA 111, 4560-4565.

Ge, J., Gong, Y.N., Xu, Y., and Shao, F. (2012). Preventing bacterial DNA release and absent in melanoma 2 inflammasome activation by a Legionella effector functioning in membrane trafficking. Proc Natl Acad Sci USA 109, 6193-6198.

Ge, J., Xu, H., Li, T., Zhou, Y., Zhang, Z., Li, S., Liu, L., and Shao, F. (2009). A Legionella type IV effector activates the NF-kappaB pathway by phosphorylating the IkappaB family of inhibitors. Proc Natl Acad Sci USA 106, 13725-13730.

Guo, Z., Stephenson, R., Qiu, J., Zheng, S., and Luo, Z.Q. (2014). A Legionella effector modulates host cytoskeletal structure by inhibiting actin polymerization. Microbes Infect 16, 225-236.

Gurcel, L., Abrami, L., Girardin, S., Tschopp, J., and van der Goot, F.G. (2006). Caspase-1 activation of lipid metabolic pathways in response to bacterial pore-forming toxins promotes cell survival. Cell 126, 1135-1145.

Hawn, T.R., Verbon, A., Janer, M., Zhao, L.P., Beutler, B., and Aderem, A. (2005). Toll-like receptor 4 polymorphisms are associated with resistance to Legionnaires' disease. Proc Natl Acad Sci USA 102, 2487-2489.

Hawn, T.R., Verbon, A., Lettinga, K.D., Zhao, L.P., Li, S.S., Laws, R.J., Skerrett, S.J., Beutler, B., Schroeder, L., Nachman, A., Ozinsky, A., Smith, K.D., and Aderem, A. (2003). A common dominant TLR5 stop codon polymorphism abolishes flagellin signaling and is associated with susceptibility to legionnaires' disease. J Exp Med 198, 1563-1572.

Horenkamp, F.A., Mukherjee, S., Alix, E., Schauder, C.M., Hubber, A.M., Roy, C.R., and Reinisch, K.M. (2014). Legionella pneumophila subversion of host vesicular transport by SidC effector proteins. Traffic 15, 488-499.

Horwitz, M.A. (1983). The Legionnaires' disease bacterium (Legionella pneumophila) inhibits phagosome-lysosome fusion in human monocytes. J Exp Med 158, 2108-2126.

Hsu, F., Luo, X., Qiu, J., Teng, Y.B., Jin, J., Smolka, M.B., Luo, Z.Q., and Mao, Y. (2014). The Legionella effector SidC defines a unique family of ubiquitin ligases important for bacterial phagosomal remodeling. Proc Natl Acad Sci USA 111, 10538-10543.

Huang, L., Boyd, D., Amyot, W.M., Hempstead, A.D., Luo, Z.Q., O'Connor, T.J., Chen, C., Machner, M., Montminy, T., and Isberg, R.R.
(2011). The E Block motif is associated with Legionella pneumophila translocated substrates. Cell Microbiol 13, 227-245.

Hubber, A., Arasaki, K., Nakatsu, F., Hardiman, C., Lambright, D., De Camilli, P., Nagai, H., and Roy, C.R. (2014). The machinery at endoplasmic reticulum-plasma membrane contact sites contributes to spatial regulation of multiple Legionella effector proteins. PLoS Pathog 10, e1004222.

Hubber, A., Kubori, T., and Nagai, H. (2013). Modulation of the ubiquitination machinery by legionella. Curr Top Microbiol Immunol 376, 227-247.

Ingmundson, A., Delprato, A., Lambright, D.G., and Roy, C.R. (2007). Legionella pneumophila proteins that regulate Rab1 membrane cycling. Nature 450, 365-369.

Isberg, R.R., O'Connor, T.J., and Heidtman, M. (2009). The Legionella pneumophila replication vacuole: making a cosy niche inside host cells. Nat Rev Microbiol 7, 13-24.

Jones, J.D., and Dangl, J.L. (2006). The plant immune system. Nature 444, 323-329.

Kofoed, E.M., and Vance, R.E. (2011). Innate immune recognition of bacterial ligands by NAIPs determines inflammasome specificity. Nature 477, 592-595.

Kubori, T., Hyakutake, A., and Nagai, H. (2008). Legionella translocates an E3 ubiquitin ligase that has multiple U-boxes with distinct functions. Mol Microbiol 67, 1307-1319.

Kumpers, P., Tiede, A., Kirschner, P., Girke, J., Ganser, A., and Peest, D. (2008). Legionnaires' disease in immunocompromised patients: a case report of Legionella longbeachae pneumonia and review of the literature. J Med Microbiol 57, 384-387.

Lamkanfi, M., and Dixit, V.M. (2014). Mechanisms and functions of inflammasomes. Cell 157, 1013-1022.

Lee, M.S., and Kim, Y.J. (2007). Pattern-recognition receptor signaling initiated from extracellular, membrane, and cytoplasmic space. Mol Cells 23, 1-10.

Li, T., Lu, Q., Wang, G., Xu, H., Huang, H., Cai, T., Kan, B., Ge, J., and Shao, F. (2013). SET-domain bacterial effectors target heterochromatin protein 1 to activate host rDNA transcription. EMBO Rep 14, 733-740.

Lifshitz, Z., Burstein, D., Peeri, M., Zusman, T., Schwartz, K., Shuman, H.A., Pupko, T., and Segal, G. (2013). Computational modeling and experimental validation of the Legionella and Coxiella virulence-related type-IVB secretion signal. Proc Natl Acad Sci USA 110, E707-715.

Lippmann, J., Muller, H.C., Naujoks, J., Tabeling, C., Shin, S., Witzenrath, M., Hellwig, K., Kirschning, C.J., Taylor, G.A., Barchet, W., Bauer, S., Suttorp, N., Roy, C.R., and Opitz, B. (2011). Dissection of a type I interferon pathway in controlling bacterial intracellular infection in mice. Cell Microbiol 13, 1668-1682.

Losick, V.P., Haenssler, E., Moy, M.Y., and Isberg, R.R. (2010). LnaB: a Legionella pneumophila activator of NF-kappaB. Cell Microbiol 12, 1083-1097.

Losick, V.P., and Isberg, R.R. (2006). NF-kappaB translocation prevents host cell death after low-dose challenge by Legionella pneumophila. J Exp Med 203, 2177-2189.

Luo, Z.Q., and Isberg, R.R. (2004). Multiple substrates of the Legionella pneumophila Dot/Icm system identified by interbacterial protein transfer. Proc Natl Acad Sci USA 101, 841-846.

Machner, M.P., and Isberg, R.R. (2006). Targeting of host Rab GTPase function by the intravacuolar pathogen Legionella pneumophila. Dev Cell 11, 47-56.

McDade, J.E., Shepard, C.C., Fraser, D.W., Tsai, T.R., Redus, M.A., and Dowdle, W.R. (1977). Legionnaires' disease: isolation of a bacterium and demonstration of its role in other respiratory disease. N Engl J Med 297, 1197-1203.

Michard, C., Sperandio, D., Bailo, N., Pizarro-Cerda, J., LeClaire, L., Chadeau-Argaud, E., Pombo-Gregoire, I., Hervet, E., Vianney, A., Gilbert, C., Faure, M., Cossart, P., and Doublet, P. (2015). The Legionella Kinase LegK2 Targets the ARP2/3 Complex To Inhibit Actin Nucleation on Phagosomes and Allow Bacterial Evasion of the Late Endocytic Pathway. mBio 6, e00354-00315.

Molofsky, A.B., Byrne, B.G., Whitfield, N.N., Madigan, C.A., Fuse, E.T., 
Tateda, K., and Swanson, M.S. (2006). Cytosolic recognition of flagellin by mouse macrophages restricts Legionella pneumophila infection. J Exp Med 203, 1093-1104.

Monroe, K.M., McWhirter, S.M., and Vance, R.E. (2009). Identification of host cytosolic sensors and bacterial factors regulating the type I interferon response to Legionella pneumophila. PLoS Pathog 5, e1000665.

Mukherjee, S., Liu, X., Arasaki, K., McDonough, J., Galan, J.E., and Roy, C.R. (2011). Modulation of Rab GTPase function by a protein phosphocholine transferase. Nature 477, 103-106.

Muller, M.P., Peters, H., Blumer, J., Blankenfeldt, W., Goody, R.S., and Itzen, A. (2010). The Legionella effector protein DrrA AMPylates the membrane traffic regulator Rab1b. Science 329, 946-949.

Murata, T., Delprato, A., Ingmundson, A., Toomre, D.K., Lambright, D.G., and Roy, C.R. (2006). The Legionella pneumophila effector protein DrrA is a Rab1 guanine nucleotide-exchange factor. Nat Cell Biol 8, 971-977.

Nagai, H., Kagan, J.C., Zhu, X., Kahn, R.A., and Roy, C.R. (2002). A bacterial guanine nucleotide exchange factor activates ARF on Legionella phagosomes. Science 295, 679-682.

Neunuebel, M.R., Chen, Y., Gaspar, A.H., Backlund, P.S., Jr., Yergey, A., and Machner, M.P. (2011). De-AMPylation of the small GTPase Rab1 by the pathogen Legionella pneumophila. Science 333, 453-456.

Odorizzi, G., Babst, M., and Emr, S.D. (2000). Phosphoinositide signaling and the regulation of membrane trafficking in yeast. Trends Biochem Sci 25, 229-235.

Ragaz, C., Pietsch, H., Urwyler, S., Tiaden, A., Weber, S.S., and Hilbi, H. (2008). The Legionella pneumophila phosphatidylinositol-4 phosphate-binding type IV substrate SidC recruits endoplasmic reticulum vesicles to a replication-permissive vacuole. Cell Microbiol 10, 2416-2433.

Rathinam, V.A., Jiang, Z., Waggoner, S.N., Sharma, S., Cole, L.E., Waggoner, L., Vanaja, S.K., Monks, B.G., Ganesan, S., Latz, E., Hornung, V., Vogel, S.N., Szomolanyi-Tsuda, E., and Fitzgerald, K.A. (2010). The AIM2 inflammasome is essential for host defense against cytosolic bacteria and DNA viruses. Nat Immunol 11, 395-402.

Ren, T., Zamboni, D.S., Roy, C.R., Dietrich, W.F., and Vance, R.E. (2006). Flagellin-deficient Legionella mutants evade caspase-1- and Naip5-mediated macrophage immunity. PLoS Pathog 2, e18.

Rolando, M., Sanulli, S., Rusniok, C., Gomez-Valero, L., Bertholet, C., Sahr, T., Margueron, R., and Buchrieser, C. (2013). Legionella pneumophila Effector RomA Uniquely Modifies Host Chromatin to Repress Gene Expression and Promote Intracellular Bacterial Replication. Cell Host Microbe 13, 395-405.

Rothmeier, E., Pfaffinger, G., Hoffmann, C., Harrison, C.F., Grabmayr, H., Repnik, U., Hannemann, M., Wolke, S., Bausch, A., Griffiths, G., Muller-Taubenberger, A., Itzen, A., and Hilbi, H. (2013). Activation of Ran GTPase by a Legionella effector promotes microtubule polymerization, pathogen vacuole motility and infection. PLoS Pathog 9, e1003598.

Shi, J., Zhao, Y., Wang, Y., Gao, W., Ding, J., Li, P., Hu, L., and Shao, F. (2014). Inflammatory caspases are innate immune receptors for intracellular LPS. Nature 514, 187-192.

Shin, S., Case, C.L., Archer, K.A., Nogueira, C.V., Kobayashi, K.S., Flavell, R.A., Roy, C.R., and Zamboni, D.S. (2008). Type IV secretion-dependent activation of host MAP kinases induces an increased proinflammatory cytokine response to Legionella pneumophila. PLoS Pathog 4, e1000220.

Simon, S., Wagner, M.A., Rothmeier, E., Muller-Taubenberger, A., and Hilbi, H. (2014). Icm/Dot-dependent inhibition of phagocyte migration by Legionella is antagonized by a translocated Ran GTPase activator. Cell Microbiol 16, 977-992.

Stone, B.J., Brier, A., and Kwaik, Y.A. (1999). The Legionella pneumophila prp locus; required during infection of macrophages and amoebae.
Microbial Pathog 27, 369-376.

Strober, W., Murray, P.J., Kitani, A., and Watanabe, T. (2006). Signalling pathways and molecular interactions of NOD1 and NOD2. Nat Rev Immunol 6, 9-20.

Stuart, L.M., Paquette, N., and Boyer, L. (2013). Effector-triggered versus pattern-triggered immunity: how animals sense pathogens. Nat Rev Immunol 13, 199-206.

Sun, L., Wu, J., Du, F., Chen, X., and Chen, Z.J. (2013). Cyclic GMP-AMP synthase is a cytosolic DNA sensor that activates the type I interferon pathway. Science 339, 786-791.

Tan, Y., Arnold, R.J., and Luo, Z.Q. (2011). Legionella pneumophila regulates the small GTPase Rab1 activity by reversible phosphorylcholination. Proc Natl Acad Sci USA 108, 21212-21217.

Tan, Y., and Luo, Z.Q. (2011). Legionella pneumophila SidD is a deAMPylase that modifies Rab1. Nature 475, 506-509.

Tilney, L.G., Harb, O.S., Connelly, P.S., Robinson, C.G., and Roy, C.R. (2001). How the parasitic bacterium Legionella pneumophila modifies its phagosome and transforms it into rough ER: implications for conversion of plasma membrane to the ER membrane. J Cell Sci 114, 4637-4650.

Toulabi, L., Wu, X., Cheng, Y., and Mao, Y. (2013). Identification and structural characterization of a Legionella phosphoinositide phosphatase. J Biol Chem 288, 24518-24527.

Turk, B., and Turk, V. (2009). Lysosomes as "suicide bags" in cell death: myth or reality? J Biol Chem 284, 21783-21787.

Viner, R., Chetrit, D., Ehrlich, M., and Segal, G. (2012). Identification of two Legionella pneumophila effectors that manipulate host phospholipids biosynthesis. PLoS Pathog 8, e1002988.

Wang, X., Parashar, K., Sitaram, A., and Bliska, J.B. (2014). The GAP Activity of Type III Effector YopE Triggers Killing of Yersinia in Macrophages. PLoS Pathog 10, e1004346.

Weber, S.S., Ragaz, C., Reus, K., Nyfeler, Y., and Hilbi, H. (2006). Legionella pneumophila exploits $\mathrm{PI}(4) \mathrm{P}$ to anchor secreted effector proteins to the replicative vacuole. PLoS Pathog 2, e46.

Woodward, J.J., Iavarone, A.T., and Portnoy, D.A. (2010). c-di-AMP secreted by intracellular Listeria monocytogenes activates a host type I interferon response. Science 328, 1703-1705.

Xu, H., Yang, J., Gao, W., Li, L., Li, P., Zhang, L., Gong, Y.N., Peng, X., Xi, J.J., Chen, S., Wang, F., and Shao, F. (2014). Innate immune sensing of bacterial modifications of Rho GTPases by the Pyrin inflammasome. Nature 513, 237-241.

Xu, L., and Luo, Z.Q. (2013). Cell biology of infection by Legionella pneumophila. Microbes Infect 15, 157-167.

Xu, L., Shen, X., Bryan, A., Banga, S., Swanson, M.S., and Luo, Z.Q. (2010). Inhibition of host vacuolar $\mathrm{H}^{+}$-ATPase activity by a Legionella pneumophila effector. PLoS Pathog 6, e1000822.

Yamamoto, Y., Klein, T.W., Newton, C.A., and Friedman, H. (1988). Interaction of Legionella pneumophila with peritoneal macrophages from various mouse strains. Adv Exp Med Biol 239, 89-98.

Zhao, Y., Yang, J., Shi, J., Gong, Y.N., Lu, Q., Xu, H., Liu, L., and Shao, F. (2011). The NLRC4 inflammasome receptors for bacterial flagellin and type III secretion apparatus. Nature 477, 596-600.

Zhu, W., Banga, S., Tan, Y., Zheng, C., Stephenson, R., Gately, J., and Luo, Z.Q. (2011). Comprehensive identification of protein substrates of the Dot/Icm type IV transporter of Legionella pneumophila. PLoS One 6, e17638.

Zhu, W., Hammad, L.A., Hsu, F., Mao, Y., and Luo, Z.Q. (2013). Induction of caspase 3 activation by multiple Legionella pneumophila Dot/Icm substrates. Cell Microbiol 15, 1783-1795.

Zhu, W., Tao, L., Quick, M.L., Joyce, J.A., Qu, J.M., and Luo, Z.Q. (2015). Sensing Cytosolic RpsL by Macrophages Induces Lysosomal Cell Death and Termination of Bacterial Infection. PLoS Pathog 11, e1004704.

Open Access This article is distributed under the terms of the Creative Commons Attribution License which permits any use, distribution, and reproduction in any medium, provided the original author(s) and source are credited. 